\title{
Inequalities for Some Functionals Associated with Bounded Linear Operators in Hilbert Spaces
}

\author{
By
}

Sever S. DragomiR*

\begin{abstract}
Some inequalities between the operator norm, numerical radius and the functionals $v_{p}, \delta_{p}$ defined in terms of the real and imaginary part of $\langle A x, x\rangle, x \in H,\|x\|=1$ are established. New upper bounds for the nonnegative quantity $\|A\|^{2}-w^{2}(A)$ with $A \in B(H)$ that complement some recent results of the author are given as well.
\end{abstract}

\section{$\S 1 . \quad$ Introduction}

Let $(H,\langle\cdot, \cdot\rangle)$ be a Hilbert space over the real or complex number field $\mathbb{K}$ $(\mathbb{K}=\mathbb{R}, \mathbb{C})$. Let $B(H)$ denote the $C^{*}$-algebra of all bounded linear operators on a complex Hilbert space $H$. For $A \in B(H)$, let $w(A)$ and $\|A\|$ denote the numerical radius and the usual operator norm of $A$, respectively. It is well known that $w(\cdot)$ defines a norm on $B(H)$ and for every $A \in B(H)$

$$
\frac{1}{2}\|A\| \leq w(A) \leq\|A\|
$$

For recent results concerning inequalities between numerical radius and operator norms, see [3], [4] and [5].

Communicated by August 01, 2006

2000 Mathematics Subject Classification(s): 47A12, 47A30, 47A63.

Key words and phrases: Numerical radius, Operator norm, Semi-inner products, Bounded linear operators, Accretive operators, $C^{*}$-Banach algebra, Reverse inequalities.

* School of Computer Science and Mathematics, Victoria University, PO Box 14428, Melbourne City, VIC, Australia. 8001.

e-mail: sever.dragomir@vu.edu.au

http://rgmia.vu.edu.au/dragomir

(c) 2007 Research Institute for Mathematical Sciences, Kyoto University. All rights reserved. 
Replacing the supremum with the infimum in the definitions of the operator norm and numerical radius, we can also consider the quantities $\ell(A):=$ $\inf _{\|x\|=1}\|A x\|$ and $m(A)=\inf _{\|x\|=1}|\langle A x, x\rangle|$. By the Schwarz inequality, it is obvious that $m(A) \leq \ell(A)$ for each $A \in B(H)$.

We can also consider the functionals $v_{s}, \delta_{s}: B(H) \rightarrow \mathbb{R}$ given by

$$
v_{s}(A):=\sup _{\|x\|=1} \operatorname{Re}\langle A x, x\rangle \quad \text { and } \quad \delta_{s}(A):=\sup _{\|x\|=1} \operatorname{Im}\langle A x, x\rangle
$$

where " $s$ " stands for supremum, while the corresponding ones for infimum are defined as:

$$
v_{i}(A):=\inf _{\|x\|=1} \operatorname{Re}\langle A x, x\rangle \quad \text { and } \quad \delta_{i}(A):=\inf _{\|x\|=1} \operatorname{Im}\langle A x, x\rangle .
$$

We notice that the functionals $v_{p}, \delta_{p}$ with $p \in\{s, i\}$ are obviously connected by the formula

$$
\delta_{p}(A)=-v_{q}(i A) \quad \text { for any } \quad A \in B(H),
$$

where $p \neq q$ and the " $i$ " in front of $A$ represents the imaginary unit. Also, by definition, $v_{s}$ and $\delta_{s}$ are positive homogeneous and subadditive while $v_{i}$ and $\delta_{i}$ are positive homogeneous and superadditive.

Due to the fact that for any $x \in H,\|x\|=1$ we have

$$
-w(A) \leq-|\langle A x, x\rangle| \leq \operatorname{Re}\langle A x, x\rangle, \operatorname{Im}\langle A x, x\rangle \leq|\operatorname{Im}\langle A x, x\rangle| \leq w(A),
$$

then, by taking the supremum and the infimum respectively over $x \in H,\|x\|=$ 1, we deduce the simple inequality:

$$
\max \left\{\left|v_{p}(A)\right|,\left|\delta_{p}(A)\right|\right\} \leq w(A), \quad A \in B(H)
$$

where $p \in\{s, i\}$.

For two operators $A, B \in B(H)$ we define

$$
w_{e}(A, B):=\sup _{\|x\|=1}\left[|\langle A x, x\rangle|^{2}+|\langle B x, x\rangle|^{2}\right]^{\frac{1}{2}}
$$

and

$$
m_{e}(A, B):=\inf _{\|x\|=1}\left[|\langle A x, x\rangle|^{2}+|\langle B x, x\rangle|^{2}\right]^{\frac{1}{2}} .
$$

$w_{e}(A, B)$ is called the Euclidean operator radius for the pair $(A, B)$ and has been introduced in [6] (see also [2]). In [6] the author considered the concept 
for $n$ operators. In the case $n=2$ and with the above notations, we can state the inequality obtained by Popescu [6]:

$$
\frac{1}{2 \sqrt{2}}\left\|A^{*} A+B^{*} B\right\|^{\frac{1}{2}} \leq w_{e}(A, B) \leq\left\|A^{*} A+B^{*} B\right\|^{\frac{1}{2}}
$$

for any $(A, B) \in B^{2}(H)$. The constant $\frac{1}{2 \sqrt{2}}$ and 1 in (1.8) are sharp. In [2] the following sharp inequalities for the Euclidean operator radius have been obtained as well:

$$
\begin{aligned}
\frac{1}{\sqrt{2}} \max \{w(B+A), w(B-A)\} & \leq w_{e}(A, B) \\
& \leq \frac{1}{\sqrt{2}}\left[w^{2}(B+A)+w^{2}(B-A)\right]^{\frac{1}{2}}
\end{aligned}
$$

$$
w_{e}^{2}(A, B) \leq \max \left\{\|A\|^{2},\|B\|^{2}\right\}+w\left(B^{*} A\right)
$$

$$
w_{e}^{2}(A, B) \leq \frac{1}{2}\left[\max \left\{\|B-A\|^{2},\|B+A\|^{2}\right\}+w\left(\left(B^{*}-A^{*}\right)(B+A)\right)\right] ;
$$

$$
w_{e}^{2}(A, B) \leq \frac{1}{2}\left[\left\|A^{*} A+B^{*} B\right\|+\left\|A^{*} A-B^{*} B\right\|\right]+w\left(B^{*} A\right) ;
$$

for any $A, B \in B(H)$.

We now recall that an operator $B: H \rightarrow H$ is called accretive if $\operatorname{Re}\langle B x, x\rangle$ $\geq 0$ for any $x \in H,\|x\|=1$. Using this concept we established in [1] amongst others the following reverse inequalities connecting the operator norm with the numerical radius:

If $\psi, \varphi \in \mathbb{K}(\mathbb{K}=\mathbb{C}, \mathbb{R}), \psi \notin\{-\varphi, \varphi\}$ and the composite operator $C_{\varphi, \psi}(A)$ $:=\left(A^{*}-\bar{\varphi} I\right)(\psi I-A)$ with $A \in B(H)$ is accretive, then

$$
(0 \leq)\|A\|-w(A) \leq \frac{1}{4} \cdot \frac{|\psi-\varphi|^{2}}{|\psi+\varphi|} .
$$

Moreover, if $\operatorname{Re}(\psi \bar{\varphi})>0$, then

$$
\frac{2 \sqrt{\operatorname{Re}(\psi \bar{\varphi})}}{|\psi+\varphi|} \leq \frac{w(A)}{\|A\|},
$$


which, in the case that $|\psi-\varphi| \leq \frac{\sqrt{3}}{2}|\psi+\varphi|$ provides a refinement of the other important inequality between the operator norm and the numerical radius, namely

$$
\frac{1}{2} \leq \frac{2 \sqrt{\operatorname{Re}(\psi \bar{\varphi})}}{|\psi+\varphi|} \leq \frac{w(A)}{\|A\|} .
$$

Also, if $\operatorname{Re}(\psi \bar{\varphi})>0$, then under the assumption that $C_{\varphi, \psi}(A)$ is accretive, we also have:

$$
(0 \leq)\|A\|^{2}-w^{2}(A) \leq\left\{\begin{array}{l}
\frac{|\psi-\varphi|^{2}}{|\psi+\varphi|}\|A\|^{2}, \\
{[|\psi+\varphi|-2 \sqrt{\operatorname{Re}(\psi \bar{\varphi})}] w(A) .}
\end{array}\right.
$$

Now, if $M \geq m>0$ and $A \in B(H)$ is such that $C_{m, M}(A)=\left(A^{*}\right.$ $-m I)(M I-A)$ is accretive or, sufficiently, $C_{m, M}(A)$ is self-adjoint and positive in the operator partial order of $B(H)$, then [1]:

$$
\begin{gathered}
(1 \leq) \frac{\|A\|}{w(A)} \leq \frac{M+m}{2 \sqrt{m M}}, \\
(0 \leq)\|A\|-w(A) \leq \frac{(\sqrt{M}-\sqrt{m})^{2}}{2 \sqrt{m M}} w(A), \\
(0 \leq)\|A\|-w(A) \leq \frac{1}{4} \cdot \frac{(M-m)^{2}}{M+m}, \\
(0 \leq)\|A\|^{2}-w^{2}(A) \leq\left(\frac{M-m}{M+m}\right)^{2}\|A\|^{2}
\end{gathered}
$$

and

$$
(0 \leq)\|A\|^{2}-w^{2}(A) \leq(\sqrt{M}-\sqrt{m})^{2} w(A) .
$$

The main aim of this paper is two fold. Firstly, some natural connections amongst the functionals $v_{p}, \delta_{p}$, the operator norm and the numerical ranges $w, m, w_{e}$ and $m_{e}$ are pointed out. Secondly, some new inequalities for operators $A \in B(H)$ for which the composite operator $C_{\gamma, \Gamma}(A)$ with $\gamma, \Gamma \in \mathbb{K}$ is assumed to be $c^{2}$-accretive with $c \in \mathbb{R}$ are also given. New upper bounds for the nonnegative quantity $\|A\|^{2}-w^{2}(A)$, which complement the ones from (1.17), (1.21) and (1.22) are obtained as well. 


\section{§2. Preliminary Results}

In the following we establish an identity connecting the numerical radius of an operator with the other functionals defined in the introduction.

Lemma 1. Let $A \in B(H)$ and $\gamma, \Gamma \in \mathbb{K}$. Then for any $x \in H,\|x\|=1$ we have the equality:

$$
\begin{aligned}
\operatorname{Re}[\langle(\Gamma I-A) x, & x\rangle\langle x,(A-\gamma I) x\rangle] \\
= & \frac{1}{4}|\Gamma-\gamma|^{2}-\left|\left\langle\left(A-\frac{\gamma+\Gamma}{2} \cdot I\right) x, x\right\rangle\right|^{2} .
\end{aligned}
$$

Proof. We use the following elementary identity for complex numbers:

$$
\operatorname{Re}(a \bar{b})=\frac{1}{4}\left[|a+b|^{2}-|a-b|^{2}\right], \quad a, b \in \mathbb{C},
$$

for the choices $a=\langle(\Gamma I-A) x, x\rangle=\Gamma-\langle A x, x\rangle$ and $b=\langle(A-\gamma I) x, x\rangle=$ $\langle A x, x\rangle-\gamma$ to get

$$
\begin{aligned}
\operatorname{Re}[\langle(\Gamma I-A) x, x\rangle & \overline{\langle(A-\gamma I) x, x\rangle}] \\
& =\frac{1}{4}\left[|\Gamma-\gamma|^{2}-|\langle 2\langle A x, x\rangle-(\gamma+\Gamma)\rangle|^{2}\right]
\end{aligned}
$$

for $x \in H,\|x\|=1$, which is clearly equivalent with (2.1).

Corollary 1. For any $A \in B(H)$ and $\gamma, \Gamma \in \mathbb{K}$ we have

$$
\inf _{\|x\|=1} \operatorname{Re}[\langle(\Gamma I-A) x, x\rangle\langle x,(A-\gamma I) x\rangle]=\frac{1}{4}|\Gamma-\gamma|^{2}-w^{2}\left(A-\frac{\gamma+\Gamma}{2} \cdot I\right)
$$

and

$$
\sup _{\|x\|=1} \operatorname{Re}[\langle(\Gamma I-A) x, x\rangle\langle x,(A-\gamma I) x\rangle]=\frac{1}{4}|\Gamma-\gamma|^{2}-m^{2}\left(A-\frac{\gamma+\Gamma}{2} \cdot I\right) .
$$

The proof is obvious from the identity (2.1) on taking the infimum and the supremum over $x \in H,\|x\|=1$, respectively.

If we denote by $S_{H}:=\{x \in H \mid\|x\|=1\}$ the unit sphere in $H$ and, for $A \in B(H), \gamma, \Gamma \in \mathbb{K}$ we define

$$
\mu(A ; \gamma, \Gamma)(x):=\operatorname{Re}[\langle(\Gamma I-A) x, x\rangle\langle x,(A-\gamma I) x\rangle], \quad x \in S_{H} ;
$$


then, on utilising the elementary properties of complex numbers we have

$$
\begin{aligned}
\mu(A ; \gamma, \Gamma)(x)=(\operatorname{Re} \Gamma-\operatorname{Re}\langle A x, x\rangle)(\operatorname{Re}\langle A x, x\rangle-\operatorname{Re} \gamma) \\
+(\operatorname{Im} \Gamma-\operatorname{Im}\langle A x, x\rangle)(\operatorname{Im}\langle A x, x\rangle-\operatorname{Im} \gamma)
\end{aligned}
$$

for any $x \in S_{H}$.

If we denote:

$$
\mu_{s(i)}(A ; \gamma, \Gamma):=\sup _{\|x\|=1}\left(\inf _{\|x\|=1}\right) \mu(A ; \gamma, \Gamma)(x)
$$

then (2.4) can be stated as:

$$
\mu_{i}(A ; \gamma, \Gamma)+w^{2}\left(A-\frac{\gamma+\Gamma}{2} \cdot I\right)=\frac{1}{4}|\Gamma-\gamma|^{2}
$$

while (2.5) can be stated as:

$$
\mu_{s}(A ; \gamma, \Gamma)+m^{2}\left(A-\frac{\gamma+\Gamma}{2} \cdot I\right)=\frac{1}{4}|\Gamma-\gamma|^{2}
$$

for any $A \in B(H)$ and $\gamma, \Gamma \in \mathbb{K}$.

Remark 1. Utilising the equality (2.6), a sufficient condition for the inequality $\mu_{i}(A ; \gamma, \Gamma) \geq 0$ or, equivalently, $w\left(A-\frac{\gamma+\Gamma}{2} \cdot I\right) \leq \frac{1}{2}|\Gamma-\gamma|$ to hold is that

$$
\operatorname{Re} \Gamma \geq \operatorname{Re}\langle A x, x\rangle \geq \operatorname{Re} \gamma \quad \text { and } \quad \operatorname{Im} \Gamma \geq \operatorname{Im}\langle A x, x\rangle \geq \operatorname{Im} \gamma
$$

for each $x \in H,\|x\|=1$.

The following identity that links the norm with the inner product also holds.

Lemma 2. Let $A \in B(H)$ and $\gamma, \Gamma \in \mathbb{K}$. The for each $x \in H,\|x\|=1$, we have the equality:

$$
\operatorname{Re}\left\langle\left(A^{*}-\bar{\gamma} I\right)(\Gamma I-A) x, x\right\rangle=\frac{1}{4}|\Gamma-\gamma|^{2}-\left\|\left(A-\frac{\gamma+\Gamma}{2} \cdot I\right) x\right\|^{2} .
$$

Proof. We utilise the simple identity in inner product spaces

$$
\operatorname{Re}\langle u-y, y-v\rangle=\frac{1}{4}\|u-v\|^{2}-\left\|y-\frac{u+v}{2}\right\|^{2}, \quad u, v, y \in H,
$$


for the choices $u=\Gamma x, y=A x, v=\gamma x$ with $x \in H,\|x\|=1$ to get $\operatorname{Re}\langle\Gamma x-A x, A x-\gamma x\rangle=\frac{1}{4}|\Gamma-\gamma|^{2}-\left\|\left(A-\frac{\gamma+\Gamma}{2} \cdot I\right) x\right\|^{2}, x \in H,\|x\|=1$, which is clearly equivalent with (2.10).

Corollary 2. For any $A \in B(H)$ and $\gamma, \Gamma \in \mathbb{K}$ we have

$$
v_{i}\left[\left(A^{*}-\bar{\gamma} I\right)(\Gamma I-A)\right]=\frac{1}{4}|\Gamma-\gamma|^{2}-\left\|A-\frac{\gamma+\Gamma}{2} \cdot I\right\|^{2}
$$

and

$$
v_{s}\left[\left(A^{*}-\bar{\gamma} I\right)(\Gamma I-A)\right]=\frac{1}{4}|\Gamma-\gamma|^{2}-\ell^{2}\left(A-\frac{\gamma+\Gamma}{2} \cdot I\right) .
$$

We recall that a bounded linear operator $T: H \rightarrow H$ is called strongly $c^{2}$-accretive (with $c \neq 0$ ) if $\operatorname{Re}\langle T y, y\rangle \geq c^{2}$ for each $y \in H,\|y\|=1$. For $c=0$, the operator is called accretive. Therefore, and for the sake of simplicity, we can call the operator $c^{2}$-accretive for $c \in \mathbb{R}$ and understand the statement in the above sense.

Utilising the identity (2.10) we can state the following characterisation result that will be useful in the sequel:

Lemma 3. For $A \in B(H)$ and $\gamma, \Gamma \in \mathbb{K}, c \in \mathbb{R}$, the following statements are equivalent:

(i) The operator $C_{\gamma, \Gamma}(A):=\left(A^{*}-\bar{\gamma} I\right)(\Gamma I-A)$ is $c^{2}$-accretive;

(ii) We have the inequality:

$$
\left\|A-\frac{\gamma+\Gamma}{2} \cdot I\right\|^{2} \leq \frac{1}{4}|\Gamma-\gamma|^{2}-c^{2}
$$

Remark 2. Since the self-adjoint operator $T: H \rightarrow H$ satisfying the condition: $T \geq c^{2} I$ in the operator partial order " $\geq$ " is $c^{2}$-accretive, then a sufficient condition for $C_{\gamma, \Gamma}(A)$ to be $c^{2}$-accretive is that $C_{\gamma, \Gamma}(A)$ is self-adjoint and $C_{\gamma, \Gamma}(A) \geq c^{2} I$.

Problem 1 (Open Problem). Characterise (give sufficient conditions for) the operator $A \in B(H)$ such that the transform $C_{\gamma, \Gamma}(A)$ is $c^{2}$-accretive for appropriate choices of $\gamma, \Gamma \in \mathbb{K}$ and $c \in \mathbb{R}$. 


\section{§3. General Inequalities}

We can state the following result that provides some inequalities between different numerical radii:

Theorem 1. For any $A \in B(H)$ and $\gamma, \Gamma \in \mathbb{K}$ we have the inequalities

$$
\frac{1}{4}|\Gamma-\gamma|^{2} \leq m^{2}\left(A-\frac{\gamma+\Gamma}{2} \cdot I\right)+\left\{\begin{array}{l}
\frac{1}{2} w_{e}^{2}(\Gamma I-A, A-\gamma I) \\
w(\Gamma I-A) w(A-\gamma I)
\end{array}\right.
$$

and

$$
\frac{1}{4}|\Gamma-\gamma|^{2} \leq w^{2}\left(A-\frac{\gamma+\Gamma}{2} \cdot I\right)+\frac{1}{2} m_{e}^{2}(\Gamma I-A, A-\gamma I) .
$$

Proof. Utilising the elementary inequality

$$
\operatorname{Re}(a \bar{b}) \leq \frac{1}{2}\left[|a|^{2}+|b|^{2}\right], \quad a, b \in \mathbb{C}
$$

we can state that

$$
\begin{aligned}
\operatorname{Re}[\langle(\Gamma I-A) x, & x\rangle \overline{\langle(A-\gamma I) x, x\rangle}] \\
& \leq \frac{1}{2}\left[|\langle(\Gamma I-A) x, x\rangle|^{2}+|\langle(A-\gamma I) x, x\rangle|^{2}\right]
\end{aligned}
$$

for any $x \in H,\|x\|=1$.

Taking the supremum over $x \in H,\|x\|=1$ in (3.4) and utilising the representation (2.5) in Corollary 1, we deduce

$$
\begin{aligned}
\frac{1}{4}|\Gamma-\gamma|^{2} & -m^{2}\left(A-\frac{\gamma+\Gamma}{2} \cdot I\right) \\
& \leq \frac{1}{2} \sup _{\|x\|=1}\left[|\langle(\Gamma I-A) x, x\rangle|^{2}+|\langle(A-\gamma I) x, x\rangle|^{2}\right] \\
& =\frac{1}{2} w_{e}^{2}(\Gamma I-A, A-\gamma I),
\end{aligned}
$$

which is clearly equivalent to the first inequality in (3.1).

Now, by the elementary inequality

$$
\operatorname{Re}(a \bar{b}) \leq|a||b| \quad \text { for each } a, b \in \mathbb{C}
$$


we can also state that

$$
\begin{aligned}
\frac{1}{4}|\Gamma-\gamma|^{2} & -m^{2}\left(A-\frac{\gamma+\Gamma}{2} \cdot I\right) \\
& \leq \sup _{\|x\|=1}[|\langle(A-\Gamma I) x, x\rangle||\langle(A-\gamma I) x, x\rangle|] \\
& \leq \sup _{\|x\|=1}|\langle(A-\Gamma I) x, x\rangle| \cdot \sup _{\|x\|=1}|\langle(A-\gamma I) x, x\rangle| \\
& =w(\Gamma I-A) w(A-\gamma I)
\end{aligned}
$$

and the second part of (3.1) is also proved.

Taking the infimum over $x \in H,\|x\|=1$ in (3.4) and making use of the representation (2.4) from Corollary 1, we deduce the inequality in (3.2).

Remark 3. If the operator $A \in B(H)$ and the complex numbers $\gamma, \Gamma$ are such that $\mu_{i}(A ; \gamma, \Gamma) \geq 0$ or, equivalently $w\left(A-\frac{\gamma+\Gamma}{2} I\right) \leq \frac{1}{2}|\Gamma-\gamma|$, then we have the reverse inequalities

$$
\begin{aligned}
0 & \leq \frac{1}{4}|\Gamma-\gamma|^{2}-m^{2}\left(A-\frac{\gamma+\Gamma}{2} \cdot I\right) \\
& \leq\left\{\begin{array}{l}
\frac{1}{2} w_{e}^{2}(\Gamma I-A, A-\gamma I) \\
w(\Gamma I-A) w(A-\gamma I)
\end{array}\right.
\end{aligned}
$$

and

$$
0 \leq \frac{1}{4}|\Gamma-\gamma|^{2}-w^{2}\left(A-\frac{\gamma+\Gamma}{2} \cdot I\right) \leq \frac{1}{2} m_{e}^{2}(\Gamma I-A, A-\gamma I)
$$

Since, in general, $w(B) \leq\|B\|, B \in B(H)$, hence a sufficient condition for (3.5) and (3.6) to hold is that $\left\|A-\frac{\gamma+\Gamma}{2} I\right\| \leq \frac{1}{2}|\Gamma-\gamma|$ holds true. We also notice that this last condition is equivalent with the fact that the operator $C_{\gamma, \Gamma}(A)=\left(A^{*}-\bar{\gamma} I\right)(\Gamma I-A)$ is accretive.

From a different perspective and as pointed out in Remark 1, a sufficient condition for $\mu_{i}(A ; \gamma, \Gamma) \geq 0$ to hold is that (2.9) holds true and, therefore, if (2.9) is valid, then both (3.5) and (3.6) can be stated.

The following reverse inequality of (3.6) is incorporated in the following result:

Proposition 1. Let $A \in B(H)$ and $\gamma, \Gamma \in \mathbb{K}$ be such that (2.9) holds 
true. Then

$$
\begin{aligned}
(0 & \leq)\left(\operatorname{Re} \Gamma-v_{s}(A)\right)\left(v_{i}(A)-\operatorname{Re} \gamma\right)+\left(\operatorname{Im} \Gamma-\delta_{s}(A)\right)\left(\delta_{i}(A)-\operatorname{Im} \gamma\right) \\
& \leq \frac{1}{4}|\Gamma-\gamma|^{2}-w^{2}\left(A-\frac{\gamma+\Gamma}{2} \cdot I\right)
\end{aligned}
$$

Proof. Taking the infimum for $x \in H,\|x\|=1$ in the identity (2.6) and utilising the representation (2.4) and the properties of infimum, we have:

$$
\begin{aligned}
& \frac{1}{4}|\Gamma-\gamma|^{2}-w^{2}\left(A-\frac{\gamma+\Gamma}{2} \cdot I\right) \\
& \geq \inf _{\|x\|=1}[(\operatorname{Re} \Gamma-\operatorname{Re}\langle A x, x\rangle)(\operatorname{Re}\langle A x, x\rangle-\operatorname{Re} \gamma)] \\
& +\inf _{\|x\|=1}[(\operatorname{Im} \Gamma-\operatorname{Im}\langle A x, x\rangle)(\operatorname{Im}\langle A x, x\rangle-\operatorname{Im} \gamma)] \\
& \geq \inf _{\|x\|=1}(\operatorname{Re} \Gamma-\operatorname{Re}\langle A x, x\rangle) \cdot \inf _{\|x\|=1}(\operatorname{Re}\langle A x, x\rangle-\operatorname{Re} \gamma) \\
& +\inf _{\|x\|=1}(\operatorname{Im} \Gamma-\operatorname{Im}\langle A x, x\rangle) \cdot \inf _{\|x\|=1}(\operatorname{Im}\langle A x, x\rangle-\operatorname{Im} \gamma) \\
& =\left(\operatorname{Re} \Gamma-\sup _{\|x\|=1} \operatorname{Re}\langle A x, x\rangle\right)\left(\inf _{\|x\|=1} \operatorname{Re}\langle A x, x\rangle-\operatorname{Re} \gamma\right) \\
& +\left(\operatorname{Im} \Gamma-\sup _{\|x\|=1} \operatorname{Im}\langle A x, x\rangle\right)\left(\inf _{\|x\|=1} \operatorname{Im}\langle A x, x\rangle-\operatorname{Im} \gamma\right)
\end{aligned}
$$

which is exactly the desired result (3.7).

The representation in Lemma 2 has its natural consequences relating the numerical values $\ell(A)$ and $w(A)$ of certain operators as described in the following:

Theorem 2. For any $A \in B(H)$ and $\gamma, \Gamma \in \mathbb{K}$ we have:

$$
\begin{aligned}
& \frac{1}{4}|\Gamma-\gamma|^{2} \leq \ell^{2}\left(A-\frac{\gamma+\Gamma}{2} \cdot I\right) \\
& +\left\{\begin{array}{l}
\frac{1}{2} w\left[\left(\bar{\Gamma} I-A^{*}\right)(\Gamma I-A)+\left(A^{*}-\bar{\gamma} I\right)(A-\gamma I)\right] \\
w\left[\left(A^{*}-\bar{\gamma} I\right)(\Gamma I-A)\right] \\
\frac{1}{4}\left\|\left(A^{*}-\bar{\gamma} I\right)(\Gamma I-A)-I\right\|^{2}
\end{array}\right.
\end{aligned}
$$


and

$$
\begin{aligned}
\frac{1}{4}|\Gamma-\gamma|^{2} \leq \| A & -\frac{\gamma+\Gamma}{2} I \|^{2} \\
& +\left\{\begin{array}{l}
\frac{1}{2} m\left[\left(\bar{\Gamma} I-A^{*}\right)(\Gamma I-A)+\left(A^{*}-\bar{\gamma} I\right)(A-\gamma I)\right] \\
m\left[\left(A^{*}-\bar{\gamma} I\right)(\Gamma I-A)\right] \\
\frac{1}{4} \ell^{2}\left[\left(A^{*}-\bar{\gamma} I\right)(\Gamma I-A)-I\right]
\end{array}\right.
\end{aligned}
$$

respectively.

Proof. Utilising the elementary inequality in inner product spaces

$$
\operatorname{Re}\langle u, v\rangle \leq \frac{1}{2}\left[\|u\|^{2}+\|v\|^{2}\right], \quad u, v \in H
$$

we can state that

$$
\begin{aligned}
& \operatorname{Re}\langle(\Gamma I-A) x,(A-\gamma I) x\rangle \\
& \leq \frac{1}{2}\left[\|(\Gamma I-A) x\|^{2}+\|(A-\gamma I) x\|^{2}\right] \\
& =\frac{1}{2}\left[\left\langle\left(\bar{\Gamma} I-A^{*}\right)(\Gamma I-A) x, x\right\rangle+\left\langle\left(A^{*}-\bar{\gamma} I\right)(A-\gamma I) x, x\right\rangle\right] \\
& =\frac{1}{2}\left\langle\left[\left(\bar{\Gamma} I-A^{*}\right)(\Gamma I-A)+\left(A^{*}-\bar{\gamma} I\right)(A-\gamma I)\right] x, x\right\rangle
\end{aligned}
$$

for each $x \in H,\|x\|=1$.

Taking the supremum in (3.11) over $x \in H,\|x\|=1$ and utilising the representation (2.13), we deduce the first inequality in (3.8).

Now, by the elementary inequality $\operatorname{Re}(a) \leq|a|, a \in \mathbb{C}$ we have

$$
\operatorname{Re}\left\langle\left(A^{*}-\bar{\gamma} I\right)(\Gamma I-A) x, x\right\rangle \leq\left|\left\langle\left(A^{*}-\bar{\gamma} I\right)(\Gamma I-A) x, x\right\rangle\right|,
$$

which provides, by taking the supremum over $x \in H,\|x\|=1$, the second inequality in (3.8).

Finally, on utilising the inequality

$$
\operatorname{Re}\langle u, v\rangle \leq \frac{1}{4}\|u-v\|^{2}, \quad u, v \in H
$$

we also have

$$
\operatorname{Re}\left\langle\left(A^{*}-\bar{\gamma} I\right)(\Gamma I-A) x, x\right\rangle \leq \frac{1}{4}\left\|\left[\left(A^{*}-\bar{\gamma} I\right)(\Gamma I-A)-I\right] x\right\|^{2}
$$


for any $x \in H,\|x\|=1$, which gives, by taking the supremum, the last part of (3.8).

The proof of (3.9) follows by the representation (2.12) in Corollary 2 and by the inequalities $(3.11)-(3.13)$ above in which we take the infimum over $x \in H,\|x\|=1$.

Corollary 3. Let $A \in B(H)$ and $\gamma, \Gamma \in \mathbb{K}$. If $C_{\gamma, \Gamma}(A)$ is accretive, then

$$
\begin{aligned}
0 \leq & \frac{1}{4}|\Gamma-\gamma|^{2}-\ell^{2}\left(A-\frac{\gamma+\Gamma}{2} \cdot I\right) \\
& \leq\left\{\begin{array}{l}
\frac{1}{2} w\left[\left(\bar{\Gamma} I-A^{*}\right)(\Gamma I-A)+\left(A^{*}-\bar{\gamma} I\right)(A-\gamma I)\right], \\
w\left[\left(A^{*}-\bar{\gamma} I\right)(\Gamma I-A)\right], \\
\frac{1}{4}\left\|\left(A^{*}-\bar{\gamma} I\right)(\Gamma I-A)-I\right\|^{2}
\end{array}\right.
\end{aligned}
$$

and

$$
\begin{aligned}
0 \leq & \frac{1}{4}|\Gamma-\gamma|^{2}-\left\|A-\frac{\gamma+\Gamma}{2} I\right\|^{2} \\
& \leq\left\{\begin{array}{l}
\frac{1}{2} m\left[\left(\bar{\Gamma} I-A^{*}\right)(\Gamma I-A)+\left(A^{*}-\bar{\gamma} I\right)(A-\gamma I)\right], \\
m\left[\left(A^{*}-\bar{\gamma} I\right)(\Gamma I-A)\right], \\
\frac{1}{4} \ell^{2}\left[\left(A^{*}-\bar{\gamma} I\right)(\Gamma I-A)-I\right]
\end{array}\right.
\end{aligned}
$$

respectively.

\section{$\S 4 . \quad$ Reverse Inequalities}

The inequality $\|A\| \geq w(A)$ for any bounded linear operator $A \in B(H)$ is a fundamental result in Operator Theory and therefore it is useful to know some upper bounds for the nonnegative quantity $\|A\|-w(A)$ under various assumptions for the operator $A$. In our recent paper [1] several such inequalities have been obtained. In order to establish some new results that would complement the inequalities outlined in the Introduction, we need the following lemma which provides two simple identities of interest: 
Lemma 4. For any $A \in B(H)$ and $\gamma, \Gamma \in \mathbb{K}$ we have

$$
\begin{aligned}
& \|A x\|^{2}-|\langle A x, x\rangle|^{2} \\
& =\left\|\left(A-\frac{\gamma+\Gamma}{2} \cdot I\right) x\right\|^{2}-\left|\left\langle\left(A-\frac{\gamma+\Gamma}{2} \cdot I\right) x, x\right\rangle\right|^{2} \\
& =\operatorname{Re}[\langle(\Gamma I-A) x, x\rangle\langle x,(A-\gamma I) x\rangle]-\operatorname{Re}\langle(\Gamma I-A) x,(A-\gamma I) x\rangle,
\end{aligned}
$$

for each $x \in H,\|x\|=1$.

Proof. The first identity is obvious by direct calculation. The second identity can be obtained, for instance, by subtracting the identity (2.10) from (2.1).

As a natural application of the above lemma in providing upper bounds for the nonnegative quantity $\|A\|^{2}-w^{2}(A), A \in B(H)$, we can state the following result:

Theorem 3. For any $A \in B(H)$ and $\gamma, \Gamma \in \mathbb{K}$ we have

$$
\begin{aligned}
(0 & \leq)\|A\|^{2}-w^{2}(A) \\
& \leq\left\|A-\frac{\gamma+\Gamma}{2} I\right\|^{2}-m^{2}\left(A-\frac{\gamma+\Gamma}{2} \cdot I\right) \\
& =\frac{1}{4}|\Gamma-\gamma|^{2}-m^{2}\left(A-\frac{\gamma+\Gamma}{2} \cdot I\right)-v_{i}\left[\left(A^{*}-\bar{\gamma} I\right)(\Gamma I-A)\right] .
\end{aligned}
$$

Proof. From the first identity in (4.1) we have

$$
\|A x\|^{2}=|\langle A x, x\rangle|^{2}+\left\|\left(A-\frac{\gamma+\Gamma}{2} \cdot I\right) x\right\|^{2}-\left|\left\langle\left(A-\frac{\gamma+\Gamma}{2} \cdot I\right) x, x\right\rangle\right|^{2}
$$

for any $x \in H,\|x\|=1$.

Taking the supremum over $x \in H,\|x\|=1$ and utilising the fact that

$$
\begin{aligned}
& \sup _{\|x\|=1}\left[|\langle A x, x\rangle|^{2}+\left\|\left(A-\frac{\gamma+\Gamma}{2} \cdot I\right) x\right\|^{2}-\left|\left\langle\left(A-\frac{\gamma+\Gamma}{2} \cdot I\right) x, x\right\rangle\right|^{2}\right] \\
& \leq \sup _{\|x\|=1}|\langle A x, x\rangle|^{2}+\sup _{\|x\|=1}\left\|\left(A-\frac{\gamma+\Gamma}{2} \cdot I\right) x\right\|^{2} \\
& -\inf _{\|x\|=1}\left|\left\langle\left(A-\frac{\gamma+\Gamma}{2} \cdot I\right) x, x\right\rangle\right|^{2} \\
& =w^{2}(A)+\left\|\left(A-\frac{\gamma+\Gamma}{2} \cdot I\right) x\right\|^{2}-m^{2}\left(A-\frac{\gamma+\Gamma}{2} \cdot I\right),
\end{aligned}
$$


we deduce the first part of (4.2).

The second part follows by the identity (2.12).

Remark 4. Utilising the inequality (3.1) in Theorem 1 we can obtain from (4.2) the following result:

$$
\begin{aligned}
(0 & \leq)\|A\|^{2}-w^{2}(A) \\
& \leq-v_{i}\left[\left(A^{*}-\bar{\gamma} I\right)(\Gamma I-A)\right]+\left\{\begin{array}{l}
\frac{1}{2} w_{e}^{2}(\Gamma I-A, A-\gamma I), \\
w(\Gamma I-A) w(A-\gamma I),
\end{array}\right.
\end{aligned}
$$

which holds true for each $A \in B(H)$ and $\gamma, \Gamma \in \mathbb{K}$.

Since $m^{2}\left(A-\frac{\gamma+\Gamma}{2} I\right) \geq 0$, hence we also have the general inequality

$$
\begin{aligned}
(0 & \leq)\|A\|^{2}-w^{2}(A) \\
& \leq \frac{1}{4}|\Gamma-\gamma|^{2}-v_{i}\left[\left(A^{*}-\bar{\gamma} I\right)(\Gamma I-A)\right],
\end{aligned}
$$

for any $A \in B(H)$ and $\gamma, \Gamma \in \mathbb{K}$.

Theorem 3 admits the following particular case that provides a simple reverse inequality for $\|A\| \geq w(A)$ under some appropriate assumptions for the operator $A$ that have been considered in the introduction and are motivated by earlier results:

Corollary 4. Let $A \in B(H)$ and $\gamma, \Gamma \in \mathbb{K}, c \in \mathbb{R}$. If the composite operator $C_{\gamma, \Gamma}(A)$ is $c^{2}$-accretive, then:

$$
\begin{aligned}
(0 & \leq)\|A\|^{2}-w^{2}(A) \\
& \leq \frac{1}{4}|\Gamma-\gamma|^{2}-c^{2}-m^{2}\left(A-\frac{\gamma+\Gamma}{2} \cdot I\right) .
\end{aligned}
$$

The proof is obvious by the first part of the inequality (4.2) and by Lemma 3 which states that $C_{\gamma, \Gamma}(A)$ is $c^{2}$-accretive if and only if the inequality $(2.11)$ holds true.

Remark 5. From (4.6) we can deduce the following reverse inequalities which are coarser, but perhaps more useful when the terms in the upper bounds 
are known:

$$
\begin{aligned}
&(0 \leq)\|A\|^{2}-w^{2}(A) \\
& \leq-c^{2}+\left\{\begin{array}{l}
\frac{1}{4}|\Gamma-\gamma|^{2}, \\
\frac{1}{2} w_{e}^{2}(\Gamma I-A, A-\gamma I), \\
w(\Gamma I-A) w(A-\gamma I) .
\end{array}\right.
\end{aligned}
$$

In particular, if $C_{\gamma, \Gamma}(A)$ is accretive, then the following inequalities that complement the results from the Introduction can be stated:

$$
\begin{aligned}
(0 & \leq)\|A\|^{2}-w^{2}(A) \\
& \leq \frac{1}{4}|\Gamma-\gamma|^{2}-m^{2}\left(A-\frac{\gamma+\Gamma}{2} \cdot I\right) \\
& \leq\left\{\begin{array}{l}
\frac{1}{4}|\Gamma-\gamma|^{2}, \\
\frac{1}{2} w_{e}^{2}(\Gamma I-A, A-\gamma I), \\
w(\Gamma I-A) w(A-\gamma I) .
\end{array}\right.
\end{aligned}
$$

Remark 6. If $N \geq n>0$ and the composite operator $C_{n, N}(A)=$ $\left(A^{*}-n I\right)(N I-A)$ is $c^{2}$-accretive or, sufficiently, self-adjoint and positive definite with the constant $c^{2} \geq 0$, then we have the inequalities:

$$
\begin{aligned}
(0 & \leq)\|A\|^{2}-w^{2}(A) \\
& \leq \frac{1}{4}(N-n)^{2}-c^{2}-m^{2}\left(A-\frac{\gamma+\Gamma}{2} \cdot I\right) \\
& \leq-c^{2}+\left\{\begin{array}{l}
\frac{1}{4}(N-n)^{2}, \\
\frac{1}{2} w_{e}^{2}(N I-A, A-n I), \\
w(N I-A) w(A-n I) .
\end{array}\right.
\end{aligned}
$$

Remark 7. If the operator $A$ on the scalars $\gamma, \Gamma$ from the statement of Corollary 4 have in addition the property that

$$
\left|\left\langle\left(A-\frac{\gamma+\Gamma}{2} \cdot I\right) x, x\right\rangle\right| \geq d \text { for each } x \in H,\|x\|=1,
$$

where $d>0$ is given, then by (4.6) we also have

$$
(0 \leq)\|A\|^{2}-w^{2}(A) \leq \frac{1}{4}|\Gamma-\gamma|^{2}-c^{2}-d^{2} .
$$


We notice that a sufficient condition for (4.10) to hold is that the operator $A-\frac{\gamma+\Gamma}{2} \cdot I$ be $d$-accretive.

Remark 8. Finally, we note that if the operator $C_{n, N}(A)$ is accretive, (or sufficiently self-adjoint and positive), then we have the following reverse inequalities that complement the results from the introduction:

$$
(0 \leq)\|A\|^{2}-w^{2}(A) \leq\left\{\begin{array}{l}
\frac{1}{4}(N-n)^{2} \\
\frac{1}{2} w_{e}^{2}(N I-A, A-n I), \\
w(N I-A) w(A-n I) .
\end{array}\right.
$$

\section{References}

[1] S. S. Dragomir, Reverse inequalities for the numerical radius of linear operators in Hilbert spaces, Bull. Austral. Math. Soc. 73 (2006), no. 2, 255-262.

[2] Some inequalities for the Euclidean operator radius of two operators in Hilbert spaces, Linear Algebra Appl. 419 (2006), no. 1, 256-264.

[3] F. Kittaneh, A numerical radius inequality and an estimate for the numerical radius of the Frobenius companion matrix, Studia Math. 158 (2003), no. 1, 11-17.

[4] , Numerical radius inequalities for Hilbert space operators, Studia Math. 168 (2005), no. $1,73-80$.

[5] J. K. Merikoski and R. Kumar, Lower bounds for the numerical radius, Linear Algebra Appl. 410 (2005), 135-142.

[6] G. Popescu, Unitary invariants in multivariable operator theory, preprint: $\langle$ Arxiv.math.0A/0410492〉. 